\title{
Trends in the number of patients linked to potential vet-shopping behavior in the United States (2014-2019)
}

\author{
Kao-Ping Chua, MD, PhD1,2*; Jeanmarie Perrone, MD³; Laurel E. Redding, VMD, PhD4; Chad M. Brummett, MD5,6; \\ Shreya Bahl, MS'; Amy S. Bohnert, PhD6

\begin{abstract}
${ }^{1}$ Department of Pediatrics, Susan B. Meister Child Health Evaluation and Research Center, University of Michigan Medical School, Ann Arbor, MI

${ }^{2}$ Department of Health Management and Policy, University of Michigan School of Public Health, Ann Arbor, MI

${ }^{3}$ Department of Emergency Medicine, Center for Addiction Medicine and Policy, Perelman School of Medicine at the University of Pennsylvania, Philadelphia, PA

${ }^{4}$ University of Pennsylvania School of Veterinary Medicine, Kennett Square, PA

5 Michigan Opioid Prescribing Engagement Network, University of Michigan Medical School, Ann Arbor, Ml

${ }^{6}$ Department of Anesthesiology, University of Michigan Medical School, Ann Arbor, MI
\end{abstract} \\ *Corresponding author: Dr. Chua (chuak@med.umich.edu)
}

https://doi.org/10.2460/ajvr.21.10.0173

\section{OBJECTIVE}

To estimate the number of patients linked to vet-shopping behavior (the solicitation of controlled substance prescriptions from multiple veterinarians for misuse) in the United States using 2014-2019 data and characterize mandates for veterinarians to examine prescription drug monitoring programs (PDMPs) before prescribing controlled substances as of April 2021.

\section{SAMPLE}

National database reporting prescription dispensing from 92\% of US pharmacies from 2014 through 2019.

\section{PROCEDURES}

The annual number of patients with dispensed prescriptions for opioid analgesics, opioid cough-and-cold medications, or benzodiazepines from $\geq 4$ veterinarians was calculated. State veterinary medical associations were contacted for information on veterinarian PDMP use mandates.

\section{RESULTS}

From 2014 through 2019, the number of patients with prescriptions for any class of controlled substances from $\geq 4$ veterinarians tripled from 935 to $2,875(+207.5 \%)$. The number of patients with opioid cough-and-cold medication prescriptions from $\geq 4$ veterinarians rose from 150 to 1,348 (+798.9\%). The corresponding number for benzodiazepines rose from 185 to 440 (+137.8\%). The corresponding number for opioid analgesics peaked at 868 in 2016 before decreasing to 733 in 2019. In April 2021, 10 states mandated veterinarians to examine PDMP records of owners or animals before prescribing controlled substances; 3 mandates excluded benzodiazepines.

\section{CLINICAL RELEVANCE}

Vet shopping in the US may be increasingly common. Mandates for veterinarians to examine PDMPs before prescribing controlled substances might facilitate detection of this behavior. However, benefits of mandates should be weighed against their potential burden on veterinarians.

\section{$\mathbf{V}$} et shopping is defined as the solicitation of controlled substance prescriptions from multiple veterinarians for the purpose of misuse. ${ }^{1}$ Preventing this behavior is an important public health goal, as diversion of controlled substances prescribed by veterinarians could contribute to the growing US epidemic of drug overdose deaths., ${ }^{2,3}$ To facilitate detection of vet shopping, some states require veterinarians to examine prescription drug monitoring program (PDMP) records of owners or animals before prescribing controlled substances. ${ }^{4}$

Existing data on the number of patients linked to vet-shopping behavior derive from surveys of veteri- narians and an older survey of state PDMPs. ${ }^{3,5,6}$ The lack of recent national data impedes assessment of the justifiability of PDMP use mandates for veterinarians. Moreover, detailed data on these mandates are lacking, including the circumstances under which the mandates apply.

This study had 2 objectives. First, using 20142019 national pharmacy dispensing data, the study team assessed trends in the annual number of patients with dispensed controlled substance prescriptions from multiple veterinarians, a potential indicator of vet shopping. Second, using information provided by state veterinary medical associations 
and PDMP administrators, the study team characterized state PDMP use mandates for veterinarians effective as of April 30, 2021.

\section{Materials and Methods}

\section{Data source}

The study team conducted a repeated cross-sectional analysis of a database (IQVIA Longitudinal Prescription Database) that reports all prescriptions dispensed from $92 \%$ of US retail pharmacies and $70 \%$ of US mail-order pharmacies from 2014 through 2019. Data were purchased from the healthcare analytics company IQVIA. During this time period, the underlying contributing pharmacies changed little.

Data included prescriber identifiers and specialty, the latter of which included a category for veterinarians. Prescriptions assigned to this category included electronic prescriptions sent by veterinarians to a pharmacy and paper prescriptions provided by veterinarians to the animal owner to be filled at a pharmacy. Data also included patient year of birth and anonymized patient identifiers. In the data, patients were the entity under whose names prescriptions were dispensed. Although patient species was not reported, the age distribution of patients with dispensed prescriptions from multiple veterinarians suggested that most were animals (see below). Data did not capture direct dispensing of medications to patients by veterinarians. Because data were deidentified, analyses were exempted from review of human subjects by the Institutional Review Board of the University of Michigan Medical School.

\section{Sample}

The sample included all dispensed prescriptions from veterinarians from 2014 through 2019 for 3 classes of controlled substances: opioid analgesics (eg, tramadol, codeine, hydrocodone, and buprenorphine formulations approved for analgesia), opioid cough-and-cold medications (eg, hydrocodone or codeine products containing medications such as guaifenesin), and benzodiazepines (eg, alprazolam and diazepam). For brevity, these classes collectively are referred to as controlled substances hereafter.

\section{Number of patients linked to potential vet-shopping behavior}

For each patient with $\geq 1$ dispensed controlled substance prescription from a veterinarian in a calendar year, the study team calculated the number of unique veterinarians accounting for these controlled substance prescriptions on the basis of the total number of unique prescriber identifiers across prescriptions. Fol-

Table 1-Dispensed controlled substance prescriptions from US veterinarians and nonveterinarians from 2014 through 2019.

\begin{tabular}{|c|c|c|c|c|c|c|}
\hline Outcome & 2014 & 2015 & 2016 & 2017 & 2018 & 2019 \\
\hline \multicolumn{7}{|l|}{$\begin{array}{l}\text { Controlled substance prescriptions } \\
\text { from veterinarians }\end{array}$} \\
\hline \multicolumn{7}{|l|}{$\begin{array}{l}\text { No. of patients with } \geq 1 \\
\text { veterinarian prescription }\end{array}$} \\
\hline Any controlled substance class & 982,313 & $1,113,805$ & $1,222,989$ & $1,208,760$ & $1,089,718$ & 985,980 \\
\hline Opioid analgesics & 518,876 & 609,712 & 664,948 & 634,708 & 539,048 & 435,338 \\
\hline $\begin{array}{l}\text { Opioid cough-and-cold } \\
\text { medications }\end{array}$ & 106,663 & 113,783 & 144,006 & 165,030 & 184,017 & 211,960 \\
\hline Benzodiazepines & 356,774 & 390,310 & 414,035 & 409,022 & 366,653 & 338,682 \\
\hline \multicolumn{7}{|l|}{$\begin{array}{l}\text { Median (25th to } 75 \text { th percentile) } \\
\text { No. of days opioids were supplied }\end{array}$} \\
\hline Opioid analgesics & $20(10-30)$ & $20(10-30)$ & $20(10-30)$ & $20(10-30)$ & $21(10-30)$ & $21(10-30)$ \\
\hline $\begin{array}{l}\text { Opioid cough-and-cold } \\
\text { medications }\end{array}$ & $18(10-30)$ & $20(10-30)$ & $22(10-30)$ & $22(10-30)$ & $24(12-30)$ & $25(13-30)$ \\
\hline Benzodiazepines & $14(7-30)$ & $14(7-30)$ & $15(7-30)$ & $15(7-30)$ & $15(7-30)$ & $15(8-30)$ \\
\hline \multicolumn{7}{|l|}{$\begin{array}{l}\text { Controlled substance prescriptions } \\
\text { from nonveterinarians }\end{array}$} \\
\hline \multicolumn{7}{|l|}{$\begin{array}{l}\text { No. of patients with } \geq 1 \\
\text { nonveterinarian prescri }\end{array}$} \\
\hline Any controlled substance classa & $313,157,005$ & $312,370,334$ & $302,143,456$ & $282,925,989$ & $255,190,143$ & $235,382,862$ \\
\hline Opioid analgesics & $204,462,657$ & $199,936,819$ & $192,303,379$ & $178,191,995$ & $158,127,646$ & $144,806,135$ \\
\hline $\begin{array}{l}\text { Opioid cough-and-cold } \\
\text { medications }\end{array}$ & $7,695,426$ & $6,947,405$ & $6,182,340$ & $5,467,784$ & $4,031,557$ & $3,297,363$ \\
\hline Benzodiazepines & $100,998,922$ & $105,486,110$ & $103,657,737$ & $99,266,210$ & $92,760,940$ & $87,279,364$ \\
\hline \multicolumn{7}{|l|}{$\begin{array}{l}\text { Median ( } 25 \text { th to } 75 \text { th percentile) } \\
\text { No. of days opiods were supplied }\end{array}$} \\
\hline Opioid analgesics & $15(5-30)$ & $15(5-30)$ & $16(5-30)$ & $20(5-30)$ & $20(5-30)$ & $20(5-30)$ \\
\hline $\begin{array}{l}\text { Opioid cough-and-cold } \\
\text { medications }\end{array}$ & $8(6-12)$ & $8(6-12)$ & $8(6-12)$ & $8(6-12)$ & $8(5-12)$ & $7(5-12)$ \\
\hline Benzodiazepines & $30(25-30)$ & $30(28-30)$ & $30(28-30)$ & $30(28-30)$ & $30(25-30)$ & $30(24-30)$ \\
\hline
\end{tabular}

aClasses of controlled substances included opioid analgesics, opioid cough-and-cold medications, or benzodiazepines. Opioid analgesics excluded injectable formulations but included buprenorphine formulations used for analgesia. Opioid cough-and-cold medications included products that combined hydrocodone or codeine with medications such as guaifenesin or phenylephrine. Benzodiazepines were limited to oral formulations. 
lowing a national quality measure assessing the receipt of opioid prescriptions from multiple prescribers, the study team calculated the annual number of patients with prescriptions for any of the 3 controlled substance classes from $\geq 4$ veterinarians. ${ }^{7}$ Analyses were repeated for each of the 3 individual classes. Sensitivity analyses used thresholds of $\geq 3$ and $\geq 5$ veterinarians.

\section{Veterinarian PDMP use mandates}

To characterize PDMP use mandates for veterinarians as of April 30, 2021, the study team emailed or called veterinary medical associations in each state and the District of Columbia. When necessary, state PDMP administrators were also contacted. Measured outcomes were the number of states mandating veterinarians to examine records of animals or owners before prescribing controlled substances in any situation. The circumstances under which use mandates applied were characterized. For context, the study team calculated the number of states with PDMP use mandates for any clinician using information published online. ${ }^{4}$ Finally, using a professional legal database (WestLaw Edge; Thomson Reuters), the study team determined whether any states implemented a PDMP use mandate for veterinarians that had been repealed by April 30, 2021.

\section{Statistical analysis}

Descriptive statistics were used to assess the annual number of dispensed controlled substance prescriptions from veterinarians and the median number of days for which those substances were supplied. For context, analyses were repeated for dispensed controlled substance prescriptions from nonveterinarians. All analyses were performed with commercially available software (SAS version 9.4; SAS Institute Inc).

\section{Results}

\section{Trends in dispensed prescriptions for controlled substance from veterinarians}

A mean of 1,110,594 (SD, 104,109) controlled substance prescriptions from veterinarians were

Table 2-Number of US patients linked to potential vet-shopping behavior from 2014 through 2019.

\begin{tabular}{|c|c|c|c|c|c|c|}
\hline Outcome & 2014 & 2015 & 2016 & 2017 & 2018 & 2019 \\
\hline \multicolumn{7}{|l|}{$\begin{array}{l}\text { No. of patients with } \geq 1 \\
\text { veterinarian prescription }\end{array}$} \\
\hline Any controlled substance class & 479,755 & 538,996 & 577,161 & 567,021 & 502,291 & 448,541 \\
\hline Opioid analgesic & 241,391 & 274,935 & 290,809 & 275,612 & 229,485 & 184,364 \\
\hline Opioid cough-and-cold medications & 49,164 & 59,902 & 72,907 & 82,865 & 90,786 & 101,749 \\
\hline Benzodiazepine & 199,151 & 215,884 & 225,762 & 219,831 & 191,707 & 171,140 \\
\hline \multicolumn{7}{|l|}{$\begin{array}{l}\text { No. of patients linked to potential } \\
\text { vet-shopping behaviora }\end{array}$} \\
\hline Any controlled substance class ${ }^{b}$ & 935 & 1,711 & 2,192 & 2,325 & 2,520 & 2,875 \\
\hline Opioid analgesics & 401 & 662 & 868 & 850 & 815 & 733 \\
\hline Opioid cough-and-cold medications & 150 & 453 & 677 & 839 & 1,041 & 1,348 \\
\hline Benzodiazepine & 185 & 274 & 335 & 375 & 381 & 440 \\
\hline $\begin{array}{l}\text { Prevalence of potential vet-shopping } \\
\text { behavior among all patientsc }\end{array}$ & 0.19 & 0.32 & 0.38 & 0.41 & 0.50 & 0.64 \\
\hline \multirow{2}{*}{\multicolumn{7}{|c|}{$\begin{array}{l}\text { No. }(\%) \text { of patients linked to potential } \\
\text { vet-shopping behavior } \\
\text { Age category }\end{array}$}} \\
\hline & & & & & & \\
\hline $0-5 y$ & $17(1.8)$ & $50(2.9)$ & $\begin{array}{r}98(4.0) \\
382(174)\end{array}$ & $113(4.9)$ & & $232(8.1)$ \\
\hline $6-11$ y & $144(15.4)$ & $264(15.4)$ & $382(17.4)$ & 457 (19.7) & $578(22.9)$ & $804(28.0)$ \\
\hline $12-17$ y & $322(34.4)$ & $758(44.3)$ & $1004(45.8)$ & $1156(49.7)$ & $1169(46.4)$ & 1115 (38.9) \\
\hline $18-25 \mathrm{y}$ & $397(42.5)$ & $525(30.7)$ & $578(26.4)$ & 463 (19.9) & $410(16.3)$ & $418(14.5)$ \\
\hline$\geq 26$ y & $54(5.8)$ & $110(6.4)$ & $140(6.4)$ & $135(5.8)$ & $146(5.8)$ & $305(10.6)$ \\
\hline Unknown & $1(0.1)$ & $4(0.2)$ & $1(0.1)$ & $1(0.0)$ & $0(0.05)$ & $1(0.0)$ \\
\hline \multicolumn{7}{|l|}{ Sex } \\
\hline Female & $413(44.2)$ & $758(44.3)$ & $988(45.1)$ & 998 (42.9) & 1050 (41.7) & $1242(43.2)$ \\
\hline Male & $508(54.3)$ & $904(52.8)$ & 1138 (51.9) & 1251 (53.8) & $1371(54.4)$ & $1553(54.0)$ \\
\hline Unknown & $14(15)$ & $49(2.9)$ & $66(3.0)$ & $76(3.3)$ & $99(3.9)$ & $80(2.8)$ \\
\hline \multicolumn{7}{|l|}{ Region of US } \\
\hline Northeast & $307(32.8)$ & 508 (29.7) & $623(28.4)$ & $662(28.5)$ & $671(26.6)$ & 767 (26.7) \\
\hline Midwest & $141(15.1)$ & $286(16.7)$ & 304 (13.9) & $298(12.8)$ & 397 (15.8) & $424(14.8)$ \\
\hline South & $361(38.6)$ & $633(37.0)$ & 809 (36.9) & $840(36.1)$ & $901(35.8)$ & 1049 (36.5) \\
\hline West & $126(13.5)$ & $284(16.6)$ & $456(20.8)$ & $525(22.6)$ & 551 (21.9) & 635 (22.1) \\
\hline
\end{tabular}

apotential vet-shopping behavior was defined as the occurrence of dispensed controlled substance prescriptions from $\geq 4$ veterinarians during the year. ${ }^{b}$ The numbers in the second through fourth rows in this section do not add up to the number in the first row for 2 reasons. First, the second through fourth rows are not mutually exclusive (eg, a patient could have opioid prescriptions from $\geq 4$ veterinarians and benzodiazepine prescriptions from $\geq 4$ veterinarians). Second, some patients did not receive prescriptions for any given medication class from $\geq 4$ veterinarians, but still received controlled substance prescriptions from $\geq 4$ veterinarians (eg., opioid prescriptions from 2 veterinarians and benzodiazepine prescriptions from 2 other veterinarians). cValues represent the percentage of all patients with $\geq 1$ dispensed controlled substance prescription from a veterinarian that were linked to potential vetshopping behavior. For example, 2,875 patients had dispensed controlled substance prescriptions of any class from $\geq 4$ veterinarians in 2019, and 448,541 had dispensed controlled substance prescriptions of any class from $\geq 1$ veterinarian $(2,875 / 448,541=0.64 \%)$. 
dispensed annually from 2014 through 2019. During this period, tramadol accounted for the highest share of opioid analgesic prescriptions (92.6\%), followed by codeine (2.9\%) and hydrocodone (1.9\%). Hydrocodone products accounted for $99.6 \%$ of opioid cough-and-cold medication prescriptions, while codeine products accounted for the other $0.4 \%$. Alprazolam and diazepam accounted for $73.5 \%$ and $19.8 \%$ of benzodiazepine prescriptions, respectively.

Opioid cough-and-cold medication dispensing doubled between 2014 and 2019, from 106,663 to 211,960 prescriptions. Opioid analgesic and benzodiazepine dispensing peaked in 2016 before declining (Table 1). The median number of days for which opioid analgesics and benzodiazepines were supplied changed little during the study period, but increased from 18 to 25 days for opioid cough-andcold medication prescriptions. The annual number of veterinarians accounting for $\geq 1$ dispensed controlled substance prescription increased from 48,086 in 2014 to 54,136 in 2019.

Between 2014 and 2019, dispensing of nonveterinarian prescriptions for opioid analgesics, opioid cough-and-cold medications, and benzodiazepines decreased by $29.2 \%, 57.2 \%$, and $13.6 \%$, respectively. Median days for which opiods were supplied increased for opioid analgesic prescriptions but changed little for the other 2 classes of controlled substances (Table 1 ).

\section{Trends in potential vet-shopping behavior}

Between 2014 and 2019, the number of patients with controlled substance prescriptions from $\geq 4$ veterinarians increased from 935 to 2,875 (+207.5\%; Table 2). Between 2014 to 2019, the number of patients with opioid cough-and-cold medication prescriptions from $\geq 4$ veterinarians increased from 150 to 1,348 (+798.9\%). For benzodiazepines, the corresponding number increased from 185 to

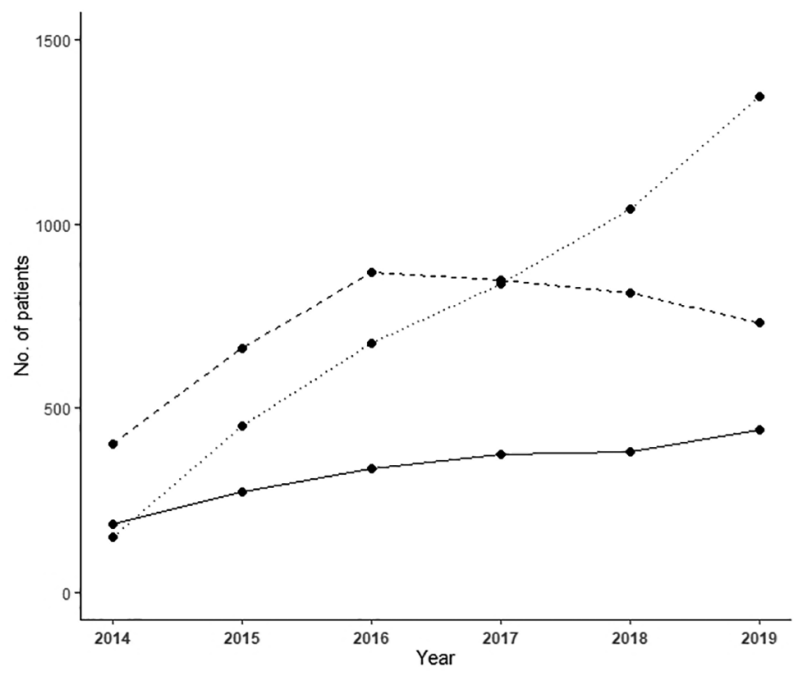

Figure 1-Temporal trends in the number of US patients with dispensed prescriptions for opioid analgesics (dashed), opioid cough-and-cold medications (dotted), and benzodiazepines (solid) from 4 or more veterinarians during the years 2014 through 2019.
$440(+137.8 \%)$. For opioid analgesics, this number peaked at 868 in 2016 before decreasing to 733 in 2019 (Figure 1). In sensitivity analyses, the number of patients with controlled substance prescriptions from $\geq 3$ and $\geq 5$ veterinarians increased from 5,460 to $11,887(+117.7 \%)$ and from 185 to $846(+357.3 \%)$ between 2014 and 2019.

Between 2014 and 2019, the number of patients with controlled substance prescriptions from $\geq 4$ veterinarians increased from 126 to $635(+404.0 \%)$ in the Western United States, the highest relative increase among the 4 regions. However, the South experienced the greatest absolute increase in the number of such patients, from 361 to 1,049 (+190.6\%). Among the 2,875 patients with controlled substance prescriptions from $\geq 4$ veterinarians in 2019, these prescriptions came from a median of 4 veterinarians (range, 4 to 42) and were dispensed a median of 28 days apart (range, 0 to 293). Among the 2,875 patients, 232 (8.1\%) were aged 0 to 5 years, 804 (28.0\%) were aged 6 to 11 years, 1,115 (38.8\%) were aged 12 to 17 years, $723(25.1 \%)$ were aged 18 years and older, and the age of 1 (0.03\%) patient was unknown.

\section{State veterinarian PDMP use mandates}

As of April 30, 2021, 49 states and the District of Columbia had a PDMP in place; Missouri was the only state that did not. A total of 47 states had PDMP use mandates before prescribing controlled substances for any clinician. Of these, 37 states exempted veterinarians from mandates and 10 did not. Of the 10 states that did not exempt veterinarians, 6 (Alaska, Connecticut, Indiana, Maine, Oklahoma, and West Virginia) required veterinarians to check PDMP records of owners in at least some instances, while 4 (Arkansas, District of Columbia, Rhode Island, and South Carolina) required veterinarians to check records of animals (Supplementary Table S1). Most PDMP use mandates for veterinarians were implemented in 2018 or afterwards. None required veterinarians to check records of both owners and animals. All 10 PDMP use mandates for veterinarians included US Drug Enforcement Administration Schedule II opioid analgesics, but 3 excluded tramadol, which is Schedule IV. ${ }^{8}$ All 10 mandates included hydrocodone cough-and-cold medications, but 3 excluded codeine cough-and-cold medications. Three state mandates excluded benzodiazepines. No states implemented a PDMP use mandate for veterinarians that had been repealed by April 30, 2021.

\section{Discussion}

Between 2014 and 2019, the number of patients with prescriptions for any class of controlled substances from $\geq 4$ veterinarians increased more than 3 -fold. The number of patients with opioid analgesic prescriptions from $\geq 4$ veterinarians declined after 2016, potentially owing to growing awareness among veterinarians of the potential for opioid misuse by animal owners, increasing availability of nonopioid treatments to manage pain in animals, or increasing evidence questioning the efficacy of tramadol for dogs. ${ }^{9}$ 
In contrast, the corresponding number for opioid cough-and-cold medications rose 9-fold, potentially because veterinarians were less aware of the potential for misuse of these medications by animal owners, allowing those engaged in misuse to obtain these medications more easily compared with opioid analgesics. Of additional concern, the median number of days supplied for opioid cough-and-cold medication prescriptions from veterinarians increased from 18 to 25 days between 2014 and 2019, suggesting that an even greater quantity of medication was potentially available for diversion. While findings suggested that vet shopping is likely still rare, this behavior may be increasingly common and more frequent than previously appreciated. For example, 1 study ${ }^{5}$ estimated that $<10$ cases of vet shopping occur annually in the US.

Mandates for veterinarians to examine PDMP records before prescribing controlled substances might improve prescribing behaviors and reduce misuse of these substances by animal owners. However, the benefits of these mandates should be balanced against their costs, including time costs for veterinarians. Implementation barriers must also be addressed to realize the potential benefits of mandates, including incompatibility of PDMPs with veterinarians' electronic medical record systems, privacy concerns among animal owners, the lack of linkages between animal and owner PDMP records in many states, and the possibility of multiple PDMP records for animals owing to inconsistent recording of an animal's name or birthdate.5,10 Policymakers must also clarify the scope and types of information made available to veterinarians during a PDMP review, develop protocols for veterinarians to detect and report suspected cases of vet shopping, and create regulations for interstate sharing of PDMP data from animals and owners. While our findings did not settle the debate regarding the justifiability of veterinarian PDMP use mandates, they suggested that addressing this debate is increasingly urgent in light of the rise in the number of patients linked to potential vet-shopping behavior.

Results of the present study also had implications for states that have already implemented PDMP use mandates for veterinarians or are considering doing so. Specifically, findings suggested that veterinarian PDMP use mandates should include all controlled substances. As of April 2021, 3 of the 10 states with such mandates excluded benzodiazepines, even though the number of patients with benzodiazepine prescriptions from multiple veterinarians is increasing. Moreover, 3 states with veterinarian PDMP use mandates excluded tramadol, the opioid analgesic most frequently prescribed by veterinarians.

The primary strength of the present study was its use of a national database that captured the vast majority of prescriptions dispensed by US pharmacies. Because of this strength, this study was able to provide the most recent national data to date on the number of patients linked to potential vet-shopping behavior. Although not the study's primary objective, this study also provided the most recent national data on controlled substance prescribing patterns by veterinarians.
In contrast, prior analyses of this prescribing have been limited to single institutions or states. ${ }^{11,12}$

The present study also had limitations. First, the database did not report patient species. Notably, however, three-quarters of patients linked to potential vet-shopping behavior in 2019 were aged 0 to 17 years. To the degree that pharmacists would be unlikely to dispense a controlled substance prescription from a veterinarian to a human child aged 0 to 17 years, this finding suggested that the majority of study patients linked to potential vet-shopping behavior in 2019 were animals. If true, analyses would suggest that state veterinarian PDMP use mandates requiring examination of animals' records should be considered. Such a requirement was included in just 4 of the 10 states with veterinarian PDMP use mandates as of April 2021.

Second, data did not capture direct dispensing of controlled substances by veterinarians. The receipt of controlled substance prescriptions from multiple veterinarians through any channel, whether pharmacy or direct dispensing, is likely greater than through pharmacy dispensing only. Additionally, the lack of data on direct dispensing precluded assessment of whether veterinarians may have shifted away from dispensing toward prescribing during the study period, for example owing to PDMP use mandates or to shortages in the types of opioids dispensed directly to patients during the study period. Unfortunately, the study team is unaware of any national database that has information on direct dispensing of medications by veterinarians and that additionally can be linked to pharmacy dispensing data. It is possible that certain state PDMP databases may contain such information if the state mandates veterinarians to report direct dispensing of controlled substances. This possibility should be explored in future research.

Third, the receipt of controlled substance prescriptions from multiple veterinarians might be less concerning if these veterinarians worked at the same practice and provided cross coverage or if controlled substances were prescribed both by an animal's primary veterinarian and a veterinarian specialist to whom the animal was referred. The degree to which this occurred is unclear, as data lacked reliable information on practice location and referral patterns. However, there was little reason to suspect that the frequency of such situations increased enough to account for the $>3$-fold increase in the number of patients linked to potential vet-shopping behavior during 2014 through 2019.

Fourth, potential vet-shopping behavior was measured based on the number of unique prescriber identifiers across all controlled substance prescriptions associated with a patient identifier. However, as alluded to previously, an animal might have multiple patient identifiers if prescriptions were dispensed under different names for the animal. The result could be either overestimation or underestimation of the number of patients linked to potential vet-shopping behavior. For example, an animal with 2 patient identifiers associated with controlled- 
substance prescriptions from $\geq 4$ veterinarians would count as 2 patients linked to potential vet-shopping behavior. In contrast, an animal with 2 patient identifiers, 1 associated with 2 veterinarians and another associated with 2 other veterinarians, would not be considered to have potential vet-shopping behavior, even though prescriptions collectively came from 4 unique veterinarians.

Finally, it was impractical and beyond the scope of this study to examine the effect of PDMP use mandates for veterinarians on controlled-substance prescribing and vet-shopping behavior. Most of these mandates were implemented in 2018 or afterward, meaning that insufficient postintervention data were available to conduct such an analysis.

In conclusion, this national study demonstrated that the number of patients linked to potential vet-shopping behavior increased $>3$-fold between 2014 and 2019. This increase was primarily driven by opioid cough-and-cold medications. Findings can inform debates regarding the justifiability and design of PDMP use mandates for veterinarians. Additionally, findings highlight the importance of educating veterinarians on strategies to prevent misuse of controlled substance prescriptions by animal owners.

\section{Acknowledgments}

Funding for the data purchase was partially provided by the Susan B. Meister Child Health Evaluation and Research Center at the University of Michigan Medical School. Further support was also provided by the Substance Abuse and Mental Health Services Administration and the University of Michigan Precision Health Initiative. Dr. Chua is supported by a career development award from the National Institute on Drug Abuse (grant No. 1K08DA048110-01) and a grant from the Benter Foundation (grant No. 2020-02). Funding sources played no role in the design of the study; the collection, analysis, and interpretation of the data; and the decision to approve publication of the finished manuscript.

Dr. Brummett serves as a consultant for Heron Therapeutics, Vertex Pharmaceuticals, and Alosa Health and has received fees for providing expert testimony. The authors declare that there were no other conflicts of interest.

Dr. Chua had full access to all of the data in the study and takes responsibility for the integrity of the data and the accuracy of the data analysis.

\section{References}

1. Vet shopping and drug diversion: a guide for veterinarians. AVMA. Accessed March 18, 2021. https://www. avma.org/sites/default/files/2019-11/Opioids_VetShopping-Drug-Diversion_Guide-for-Veterinarians_flyer. pdf

2. Provisional drug overdose death counts. CDC. Accessed November 19, 2021. https://www.cdc.gov/nchs/ nvss/vsrr/drug-overdose-data.htm

3. Mason DS, Tenney L, Hellyer PW, Newman LS. Prescription opioid epidemic: do veterinarians have a dog in the fight? Am J Public Health. 2018:108(9):1162-1163. doi:10.2105/AJPH.2018.304603

4. State PDMP profiles and contacts. Prescription Drug Monitoring Program Training and Technical Assistance Center. Accessed March 31, 2021. https://www.pdmpassist.org/ State

5. Simpson RJ. Prescription drug monitoring programs: applying a one size fits all approach to human and veterinary medical professionals, custom tailoring is needed. J Anim Environ Law. 2014;5(1):1-43.

6. Patel FC, Raines JA, Kim RW, et al. Veterinarians' attitudes and practices regarding opioid-related vet shopping practices in tri-state Appalachian counties: an exploratory study. BMC Vet Res. 2020;16(1):210. doi:10.1186/ s12917-020-02428-x

7. NFQ \#2950: use of opioids from multiple providers in persons without cancer. National Quality Forum. Accessed March 1, 2021. https://www.qualityforum.org

8. Controlled substances by CSA schedule. US Drug Enforcement Agency. Accessed November 19, 2021. https:// www.deadiversion.usdoj.gov/schedules/orangebook/e_ cs sched.pdf

9. Budsberg SC, Torres BT, Kleine SA, Sandberg GS, Berjeski AK. Lack of effectiveness of tramadol hydrochloride for the treatment of pain and joint dysfunction in dogs with chronic osteoarthritis. J Am Vet Med Assoc. 2018;252(4):427-432.

10. Philbrick A. Pet prescriptions. National Community Pharmacists Association. Accessed May 1, 2021. www.ncpa. co/issues/APDEC15-Pet_Prescriptions.pdf

11. Hendricks B, Rudisill T, Pesarsick J, et al. Prescription drug monitoring program policy reform: human and veterinary practitioner prescribing in West Virginia, 2008-2020. J Public Health Policy. 2021;42(2):249-257.

12. Clarke DL, Drobatz KJ, Korzekwa C, Nelson LS, Perrone J. Trends in opioid prescribing and dispensing by veterinarians in Pennsylvania. JAMA Netw Open. 2019;2(1):e186950. doi:10.1001/jamanetworkopen.2018.6950

\section{Supplementary Materials}

Supplementary materials are available online at the journal website: avmajournals.avma.org 